\title{
ANGIOGRAFIA POR RESSONÂNCIA MAGNÉTICA NA AVALIAC̣Ão DAS ARTÉRIAS RENAIS: ACHADOS DE IMAGEM*
}

\author{
Marcelo Souto Nacif ${ }^{1}$, Alair Augusto Sarmet Moreira Damas dos Santos ${ }^{2}$, Edson Marchiori ${ }^{3}$
}

Resumo OBJETIVO: Descrever as indicações, os principais diagnósticos e os achados de imagem nas angiografias por ressonância magnética das artérias renais. MATERIAIS E MÉTODOS: Estudo retrospectivo, no período de 6/12/2001 a 11/3/2004, num total de 56 exames, totalizando 111 artérias renais estudadas. Os exames foram realizados em um equipamento de 1,5 tesla, segundo o protocolo do Serviço. RESULTADOS: Foi demonstrado que $55,4 \%(n=31)$ pacientes eram masculinos e $44,6 \%(n=25)$, femininos. 0 paciente mais novo tinha 12 anos e o mais velho, 88 anos. De um total de 25 diferentes indicações, a hipertensão arterial sistêmica com $26,7 \%$ ( $n=15$ ) foi a principal, seguida de dor abdominal e/ou lombar com 12,5\% (n $=7)$, aneurisma da aorta abdominal com $10,7 \%(n=6)$, estenose da artéria renal com $8,9 \%(n=5)$, e outros. No que se refere às imagens, $43(76,7 \%)$ exames tiveram algum tipo de alteração e $13(23,2 \%)$ foram normais. Dentre os que tinham alterações, a maioria se deu no calibre, e dentre elas, as irregularidades parietais, aneurismas e estenoses foram as mais comuns. Na artéria renal direita as alterações mais comuns foram as irregularidades parietais com $17,87 \%(n=10)$, e na artéria renal esquerda foram as estenoses com $25,45 \%$ ( $n=14$ ). CONCLUSÃO: A angio-RM mostrou-se excelente método no estudo das artérias renais, devido à sua sensibilidade e capacidade multiplanar para avaliar as estruturas vasculares.

Unitermos: Ressonância magnética; Angiografia por ressonância magnética; Artérias renais.

Abstract Magnetic resonance angiography in the evaluation of renal arteries: imaging findings.

OBJECTIVE: To describe indications, main findings and diagnosis of magnetic resonance angiographies of renal arteries. MATERIALS AND METHODS: A retrospective study including 56 imaging studies covering a total of 111 renal arteries, performed during the period between December 6, 2001 and March 11, 2004. The angiographies were performed in a $1.5 \mathrm{~T}$ scanner, in compliance with the Department protocol. RESULTS: As regards sex, it was found that $55.4 \%(n=31)$ patients were male and $44.6 \%(n=25)$ were female. The youngest patient was 12 years old and the oldest 88 years old. From a total of 25 different clinical indications, systemic arterial hypertension was the principal one with $26.7 \%(n=15)$, followed by abdominal and/or lumbar pain with $12.5 \%(n=7)$, abdominal aortic aneurysm with $10.7 \%(n=6)$, renal artery stenosis with $8.9 \%(n=5)$, and others. Among these 56 studies, $43(76.7 \%)$ had different types of findings and $13(23.2 \%)$ were normal. The majority of findings were related to vascular diameter and amongst them, parietal irregularities, aneurysms and stenosis were the most frequent. Parietal irregularity was the most frequent alteration in the right renal artery, with $17.87 \%(\mathrm{n}=10)$, and stenosis was the most frequent alteration in the left renal artery, with $25.45 \%(n=14)$. CONCLUSION: Magnetic resonance angiography has shown to be an excellent non-invasive method for evaluation of renal arteries, because of its sensitivity and multiplanar capacity for demonstrating vascular structures.

Keywords: Magnetic resonance imaging; Magnetic resonance angiography; Renal arteries.

* Trabalho realizado no Hospital de Clínicas de Niterói, Niterói, RJ, e no Departamento de Radiologia da Faculdade de Medicina da Universidade Federal do Rio de Janeiro/Hospital Universitário Clementino Fraga Filho, Rio de Janeiro, RJ.

1. Professor de Radiologia da Faculdade de Medicina de Teresópolis (CCBM-FESO) e do Curso de Especialização em Radiologia do Instituto de Pós-Graduação Médica Carlos Chagas (VOTImagem), Mestre em Radiologia pela Universidade Federal do Rio de Janeiro.

2. Professor Titular do Curso de Especialização em Radiologia do Instituto de Pós-Graduação Médica Carlos Chagas (VOTImagem), Professor Adjunto e Chefe do Serviço de Radiologia do Hospital Universitário Antônio Pedro da Universidade Federal Fluminense.

3. Professor Titular e Chefe do Departamento de Radiologia da Universidade Federal Fluminense, Coordenador Adjunto do Curso de Pós-graduação em Radiologia da Universidade Federal do Rio de Janeiro.

Endereço para correspondência: Prof. Dr. Marcelo Souto Nacif. Rua Álvares de Azevedo, 130/704, BI. A, Icaraí. Niterói. RJ, 24220-021. E-mail: msnacif@yahoo.com.br

Recebido para publicação em 12/9/2005. Aceito, após revisão, em 23/11/2005.

\section{INTRODUÇÃO}

A angiografia por ressonância magnética (angio-RM) é um método seguro e sensível na avaliação das artérias renais, com as vantagens de não se usar meio de contraste iodado e potencialmente alérgeno, não usar radiação ionizante ou os riscos de uma cateterização.

Sabendo-se que, na grande maioria das doenças que acometem a vascularização renal, as lesões se localizam nos ramos principais da artéria renal, a utilização da angio-RM torna-seuma prática valiosa. Dessa forma, após os ganhos recentes com a moderna técnica tridimensional com o uso do meio de contraste venoso, a angioRM tornou-se um método excelente mesmo nos centros com médicos experientes em ultra-sonografia (US) e tomografia computadorizada $(\mathrm{TC})^{(\mathbf{1 - 4})}$.

A estenose da artéria renal é a lesão de maior relevância, promovendo isquemia do parênquima e uma elevação da pressão arterial sistêmica de difícil controle medicamentoso. Com o passar do tempo, a estenose evolui para oclusão e permanente redução da função renal. A importância da hipertensão como problema de saúde pública e a consequiência devastadora que 
ocorre para um paciente quando em insuficiência renal têm direcionado vários estudos para que se consiga um método seguro, rápido, não invasivo e mais barato que a angiografia por cateterismo para o diagnóstico precoce dessa doença ${ }^{(2,5-8)}$.

O objetivo deste estudo foi descrever as indicações, os principais diagnósticos e os achados de imagem na angio-RM das artérias renais.

\section{MATERIAIS E MÉTODOS}

Foi realizado estudo observacional, descritivo e retrospectivo no Serviço de Radiologia do Hospital das Clínicas de Niterói, na cidade de Niterói, RJ. Foram selecionadas todas as angio-RM das artérias renais realizadas no período de 6/12/2001 a 11/3/ 2004, num total de 56 exames, o que veio a ser a nossa amostra.

Os exames foram feitos em um equipamento de 1,5 tesla, modelo Magnetom Symphony, da Siemens, com bobina de corpo, segundo o protocolo abaixo, visando às seguintes sequiências:

1 - Sequiência localizadora, gradiente eco em apnéia, ponderada em T1 nos três planos.

2 - Sequiência gradiente eco em apnéia, ponderada em T1 2D para topografar as artérias.

3 - Sequiência gradiente eco T1 3D coronal. Esta é uma mascara para subtração com a angio-RM.

4 - Teste "bolus", axial ou coronal, gradiente eco T1 2D, com baixa resolução, demonstrando uma imagem por segundo.

5 - Angio-RM com gadolínio, gradiente eco T1 3D coronal. (TR: 4,5-5,3 ms; TE: 1,9-2,1 ms; FOV: 260-300; matriz: 256-512).

6 - Gradiente eco T1 3D axial com supressão de gordura.

7 - Reconstrução arterial (MIP e MPR).

Adicional (se necessário):

8 - Seqüência turbo spin eco T2, com supressão de gordura axial em apnéia, também para relações anatômicas.

9 - Seqüência sagital T2 em apnéia (HASTE) direita e esquerda para as relações anatômicas.

10 - Seqüência tardia para estudo venoso e pielográfico.
11 - Reconstruções: venosa e do sistema coletor

Para que se obtivesse a melhor qualidade possível, antes da realização do exame era explicado ao paciente o procedimento a que ele iria ser submetido, sendo a sua compreensão e colaboração muito importantes.

O exame era realizado com o paciente em decúbito dorsal, em posição estática, instruído para que prendesse a respiração, quando necessário, e não executasse, por menor que fosse, nenhum movimento durante as aquisições das imagens.

Os dados obtidos foram reconstruídos, obtendo-se imagens bidimensionais multiplanares ou imagens tridimensionais pelas técnicas projeção de intensidade máxima ("maximum intensity projection" - MIP) ou reconstruções multiplanares ("multiple planar reconstruction" - MPR).

O tempo para a realização do exame de angio-RM foi aproximadamente o mesmo empregado em um exame de RM do abdome, cerca de dez minutos.

Para a análise dos exames os parâmetros utilizados foram o calibre (normal, irregularidades parietais, estenoses e aneurismas), o trajeto (normal ou tortuoso) e o fluxo (normal, reduzido ou ausente). Foi feita análise da aorta e das artérias renais direita e esquerda.

Para a análise dos percentuais de estenose foram utilizados os seguintes parâmetros: (A) menor que 50\%, (B) maior do que $50 \%$ e menor do que $75 \%$, (C) maior do que $75 \%$ e menor do que $100 \%$ e (D) completa (100\%). As estenoses foram, também, localizadas em terço proximal, médio e distal.

Os rins foram analisados observando-se o contorno (regular ou irregular), o volume (normal, aumentado ou reduzido) e o nefrograma (homogêneo, ausente ou heterogêneo).

Todos os exames foram revisados por pelo menos dois radiologistas do Serviço.

Em todos os exames foi utilizado meio de contraste paramagnético.

O projeto de pesquisa do presente estudo foi aprovado no Comitê de Ética em Pesquisa da Faculdade de Medicina/Hospital Universitário Antônio Pedro da Universidade Federal Fluminense, sob o número 004/05.

\section{RESULTADOS}

\section{Considerações epidemiológicas}

Dos 56 pacientes selecionados, $55,4 \%$ $(\mathrm{n}=31)$ eram do sexo masculino e $44,6 \%$ $(\mathrm{n}=25)$ eram do sexo feminino. Quanto à faixa etária, o intervalo entre 40 e 49 anos, com 12 pacientes, foi o mais prevalente, seguido pelo intervalo de 70 a 79 anos. O paciente mais novo tinha 12 anos e o mais velho tinha 88 anos, com idade média de 55,32 anos.

As indicações clínicas para a realização dos exames foram variadas. De um total de 25 indicações diferentes nos 56 exames realizados, a hipertensão arterial sistêmica foi a principal com $26,7 \%$ ( $\mathrm{n}=$ 15), seguida de dor abdominal com $12,5 \%$ $(\mathrm{n}=7)$, aneurisma da aorta abdominal com $10,7 \%(\mathrm{n}=6)$ e estenose da artéria renal com $8,9 \%(n=5)$.

\section{Considerações de imagem}

Dos 56 exames avaliados, 23,2\% ( $\mathrm{n}=$ 13) eram normais e $76,7 \%(n=43)$ mostravam algum tipo de alteração.

\section{AORTA}

De um total de 56 aortas estudadas, no que se refere ao calibre, foram encontradas 25 aortas $(44,66 \%)$ com irregularidades parietais, 20 aortas $(35,71 \%)$ de calibre normal, dez aneurismas $(17,85 \%)$, sendo que $3,57 \%(\mathrm{n}=2)$ envolviam as artérias renais e $14,26 \%(n=8)$ não as envolviam (Figura 1), e um caso $(1,78 \%$ ) de estenose (Figura 2).

$\mathrm{O}$ estudo do trajeto da aorta mostrou que existiam $67,85 \%(n=38)$ de trajetos normais e $32,15 \%(n=18)$ de trajetos tortuosos.

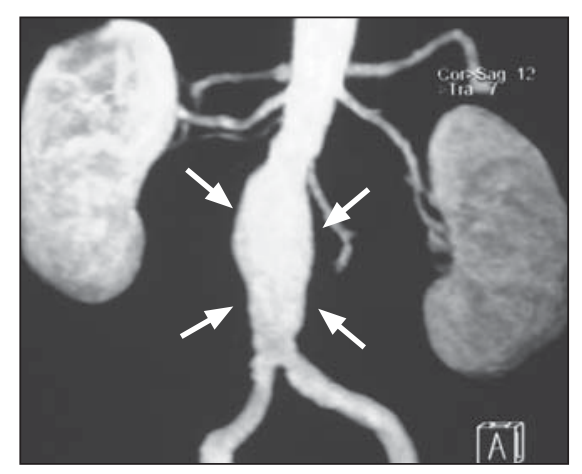

Figura 1. Aneurisma da aorta abdominal infra-renal, que não envolve as artérias renais (setas). 


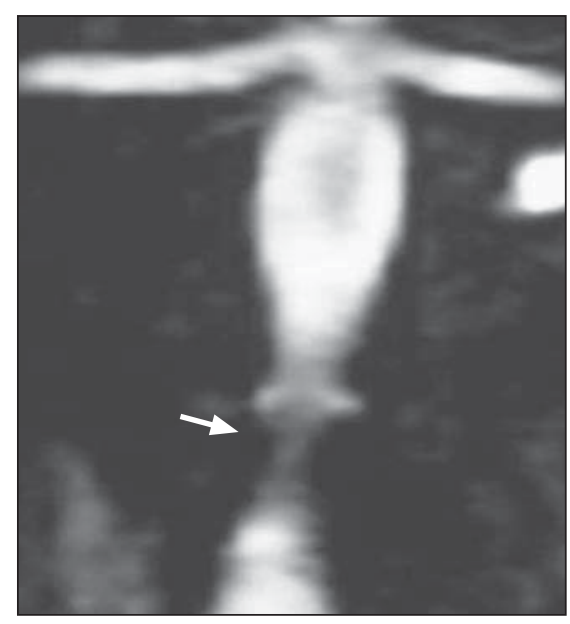

Figura 2. Estenose aórtica (seta). Doença de Takayasu.

O fluxo no interior da aorta se encontrava presente em $100 \%(n=56)$ dos exames realizados.

\section{ARTÉRIA RENAL DIREITA}

$\mathrm{Na}$ análise do calibre das 56 artérias renais direitas, $67,85 \%(\mathrm{n}=38)$ estavam com o calibre normal, $17,87 \%(\mathrm{n}=10)$ tinham irregularidades parietais (Figura 3), 14,28\% $(\mathrm{n}=8)$ apresentavam estenoses e nenhum aneurisma foi detectado.

Em relação ao trajeto, foi possível a análise em 91,07\% ( $\mathrm{n}=51)$ dos casos, e todos estavam normais. Em 8,93\% $(n=5)$ exames este estudo não foi possível pela presença de estenoses completas.

No que se refere ao fluxo, este estava normal em $85,71 \%(n=48)$ dos casos, em $5,35 \%(\mathrm{n}=3)$ estava reduzido e em $8,94 \%$ $(n=5)$ era ausente.

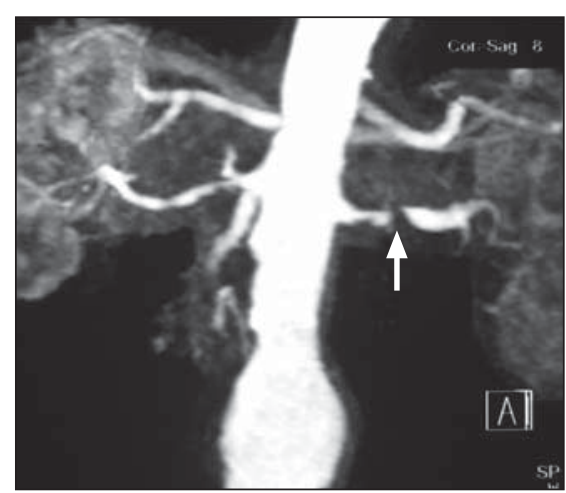

Figura 3. Irregularidades parietais na artéria renal direita associadas a estenose maior que $75 \%$ do terço médio da artéria renal esquerda (seta) e a um aneurisma infra-renal.

\section{ARTÉRIA RENAL ESQUERDA}

Foi possível a análise de 55 artérias renais esquerdas, visto que um paciente era portador de agenesia renal deste lado. Em relação ao calibre, $56,36 \%(\mathrm{n}=31)$ eram normais, $25,45 \%(\mathrm{n}=14)$ tinham estenoses, $18,19 \%(\mathrm{n}=10)$ apresentavam irregularidades parietais, e nenhum aneurisma foi identificado.

Os trajetos de $83,65 \%$ das artérias renais esquerdas $(n=46)$ eram normais, em $3,63 \%(n=2)$ eram tortuosos e em $12,72 \%$ $(\mathrm{n}=7)$ não foi possível a análise, devido à presença de estenoses completas que não permitiram a passagem do meio de contraste, não se obtendo sinal de fluxo detectável na angio-RM. O fluxo em 74,54\% (n $=41$ ) dos casos era normal, em 12,73\% (n $=7)$ era reduzido, e em $12,73 \%(n=7)$ estava ausente.

\section{ESTENOSES DAS ARTÉRIAS RENAIS}

Das 111 artérias renais estudadas, 22 apresentaram estenoses. Destas, 25,45\% $(\mathrm{n}=14)$ eram à esquerda e $14,28 \%(\mathrm{n}=8)$, à direita. No que se refere à topografia, $59,09 \%(n=13)$ localizavam-se no terço proximal das artérias renais, $31,81 \%(\mathrm{n}=$ 7) situavam-se no terço médio e 9,10\% (n = 2) localizavam-se no terço distal.

Em relação ao percentual de estenose, em $50 \%(n=11)$ a estenose era de $100 \%$, em $27,27 \%(n=6)$ as estenoses eram maiores do que $50 \%$ e menores do que $75 \%$, em $18,18 \%(n=4)$ as estenoses eram maiores do que $75 \%$ e menores do que $100 \%$ e em apenas $4,55 \%(n=1)$ as estenoses eram menores do que 50\%. Associando-se o percentual de estenose com a topografia, o achado mais freqüente foi a estenose completa (tipo D) no terço proximal com $31,81 \%(n=7)$, seguido por estenose completa no terço médio com $18,18 \%(\mathrm{n}=4)$.

\section{RIM DIREITO}

Do total de 56 rins direitos analisados, $82,14 \%(n=46)$ tinham contornos regulares e $14,86 \%(n=10)$, contornos irregulares (Figura 4). Em relação ao volume renal, $82,14 \%(n=46)$ eram normais, $12,51 \%(n$ $=7$ ) encontravam-se reduzidos e 5,35\% (n $=3$ ) estavam aumentados. No que se refere ao nefrograma, $87,50 \%(\mathrm{n}=49)$ estavam homogêneos, $10,72 \%(n=6)$ eram heterogêneos e em 1,78\% (n=1) era ausente.

\section{RIM ESQUERDO}

Do total de 55 rins esquerdos estudados, $81,81 \%(n=45)$ tinham contornos regulares e $18,19 \%(n=10)$, contornos irregulares. Na análise dos volumes renais, $87,27 \%$ $(n=48)$ eram normais, $12,72 \%(n=7)$ estavam aumentados e nenhum estava reduzido. No que se refere ao nefrograma, $85,45 \%(n=47)$ estavam homogêneos, $12,74 \%(n=7)$ eram heterogêneos (Figura 5) e em $1,81 \%(n=1)$ era ausente.

\section{OUTROS ACHADOS}

O estudo dos 56 exames também evidenciou $14,28 \%(n=8)$ de artérias renais acessórias, sendo $7,15 \%(\mathrm{n}=4)$ para o pólo inferior do rim esquerdo, 5,35\% ( $\mathrm{n}=3)$ para o pólo inferior do rim direito (Figura 6) e $1,78 \%(n=1)$ para o pólo superior do rim direito.
Figura 4. Rim direito aumentado de volume, com os contornos irregulares e nefrograma heterogêneo. 0 rim esquerdo encontra-se reduzido de volume, devido a estenose (seta) maior do que $75 \%$ de sua artéria, que está mal individualizada pelo artefato do fluxo venoso.

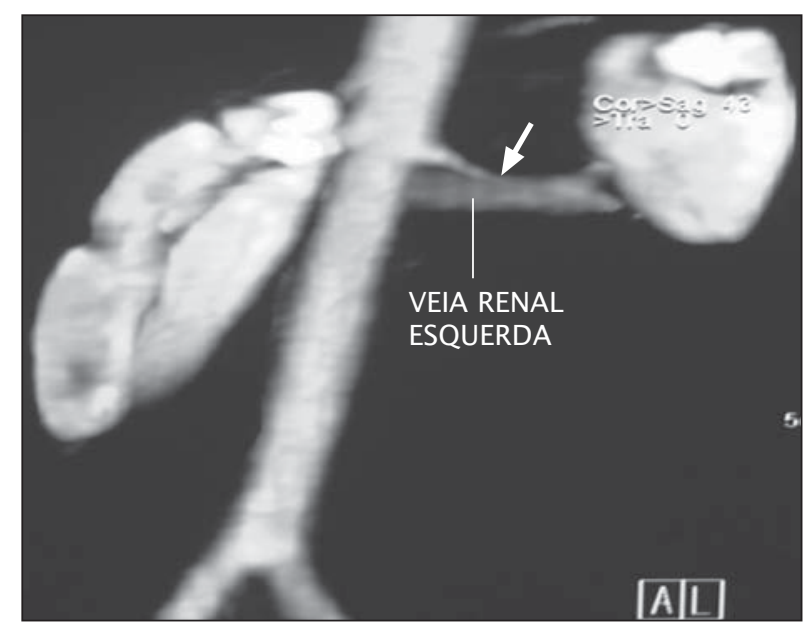


$\mathrm{Na}$ análise dos outros achados, observou-se a presença de 13 cistos renais simples, sendo sete à direita e seis à esquerda, e um caso de massa no pólo superior do rim direito, dissecção da aorta com baixo fluxo

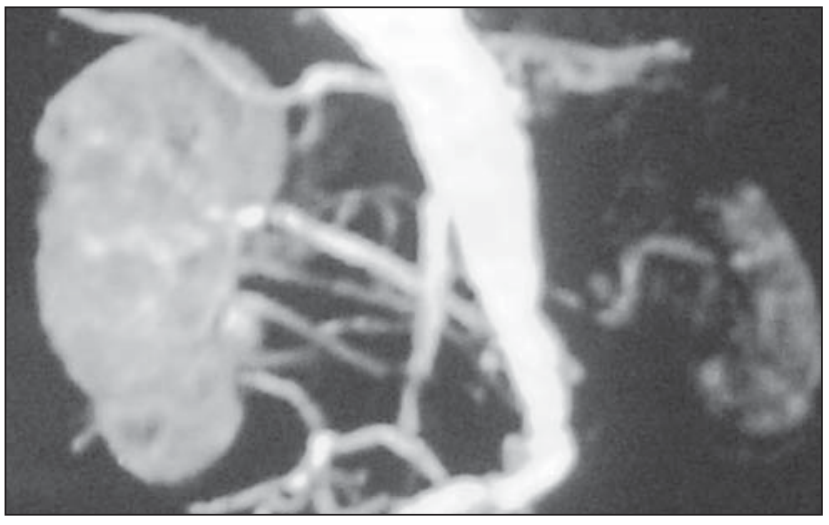

Figura 5. Rim esquerdo reduzido de volume, de contornos irregulares e nefrograma heterogêneo. A perfusão renal esquerda está reduzida por uma estenose no terço médio de sua artéria. Rim direito vicariante com nefrograma homogêneo. Observar as artérias renais acessórias direitas.

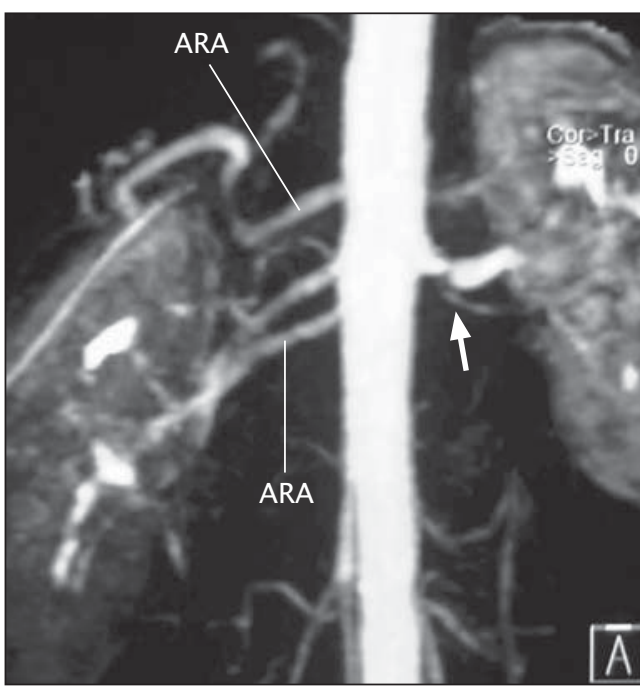

Figura 6. Artérias renais acessórias (ARA) para o pólo superior e inferior do rim direito. Estenose (seta) maior que $50 \%$ com dilatação pós-estenótica no terço proximal da artéria renal esquerda.

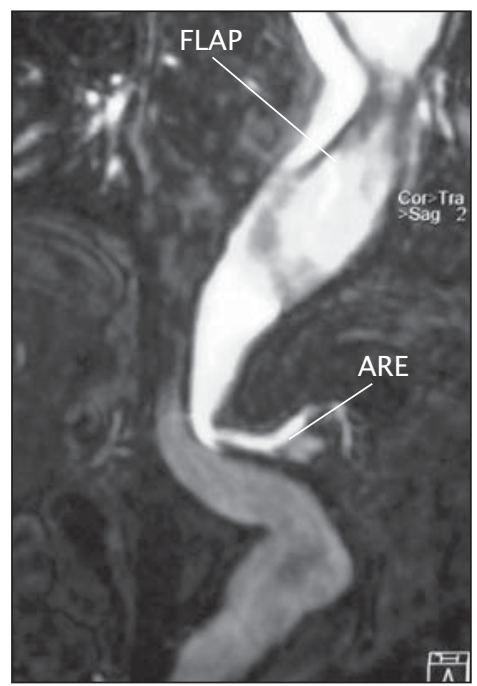

Figura 7. Dissecção aórtica (FLAP) com baixo fluxo na artéria renal direita, não individualizada. Identifica-se a artéria renal esquerda (ARE) com fluxo preservado.

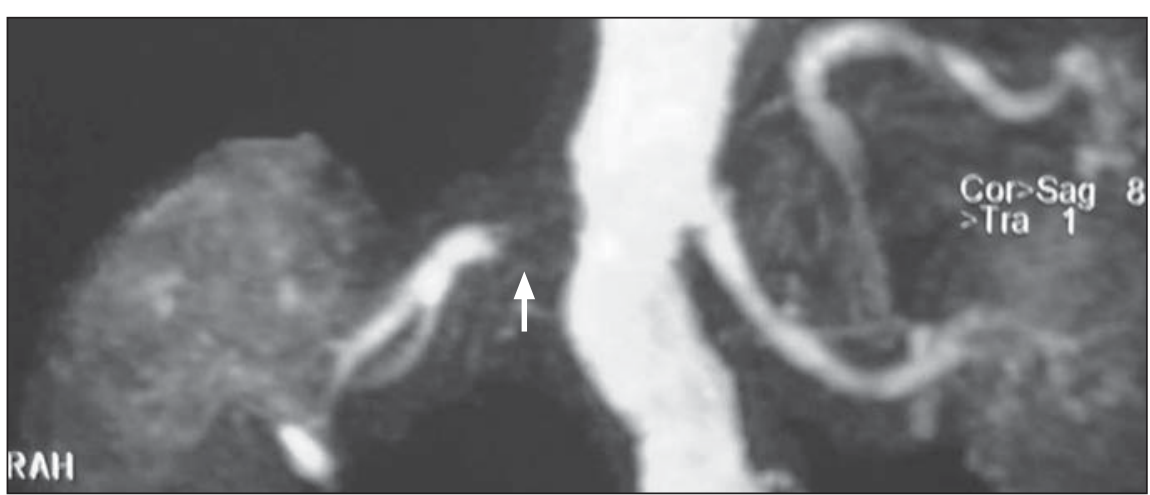

Figura 8. Endoprótese metálica na artéria renal direita (seta). 0 fluxo e a perfusão renal estão normais. gura 10), rim único à direita (Figura 11) e bifurcação da artéria renal direita no seu terço médio (Figura 12).

\section{DISCUSSÃO}

\section{Considerações clínicas e epidemiológicas}

Dos 56 pacientes selecionados, $55,40 \%$ ( $n=31$ ) eram do sexo masculino e $44,60 \%$ $(\mathrm{n}=25)$ eram do sexo feminino. Observouse que a hipertensão arterial sistêmica foi a principal indicação clínica para realização da angio-RM e, dessa forma, a pequena diferença percentual em relação ao sexo predominante se deve ao fato de que a hipertensão arterial sistêmica é uma doença que acomete ambos os sexos, sem predileção importante por nenhum deles ${ }^{(\mathbf{9 - 1 2})}$.

Quanto à faixa etária, houve prevalência na quinta década, com $21,42 \%(n=12)$ dos exames, e na oitava década, com $17,86 \%(\mathrm{n}=10)$. Notou-se, contudo, distribuição homogênea entre a quarta, sexta, sétima e nona décadas.

Sabendo-se que mais da metade dos pacientes com hipertensão arterial sistêmica teve alterações relacionadas a irregularidades parietais, e sendo estas um fator precursor das estenoses arteriais, observase que, isoladamente, é uma boa indicação para a realização deste exame, visto ter sido a indicação responsável pelo maior número de resultados alterados dentre os exames deste estudo.

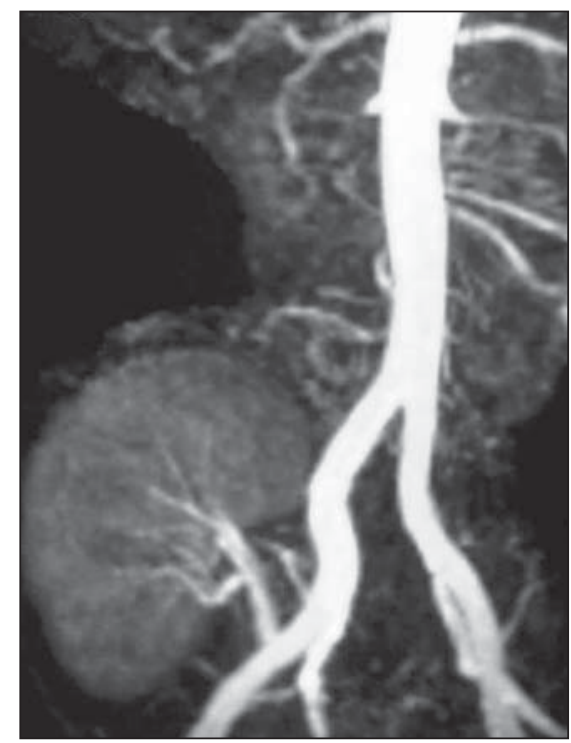

Figura 9. Rim transplantado pélvico à direita. 


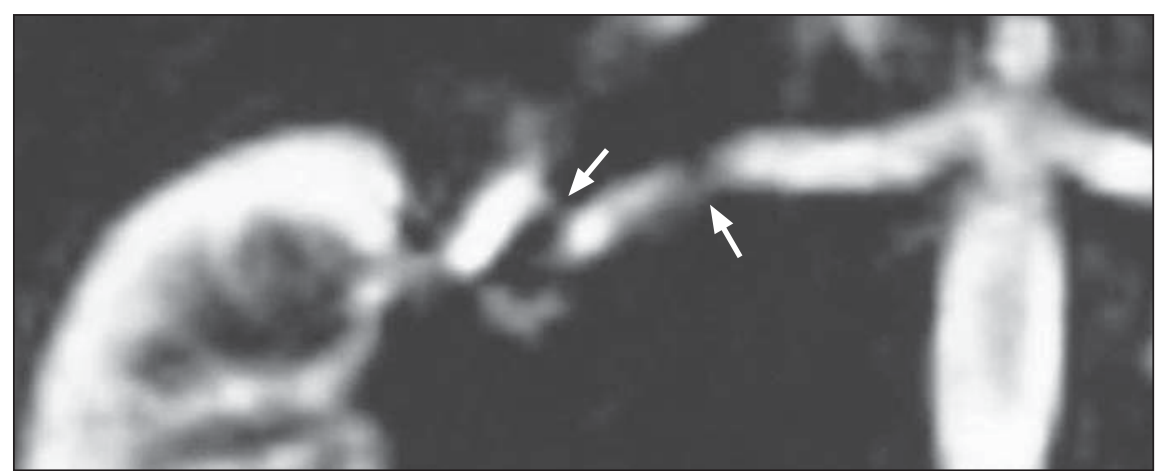

Figura 10. Artefato de fluxo (setas) simulando estenose na artéria renal direita.

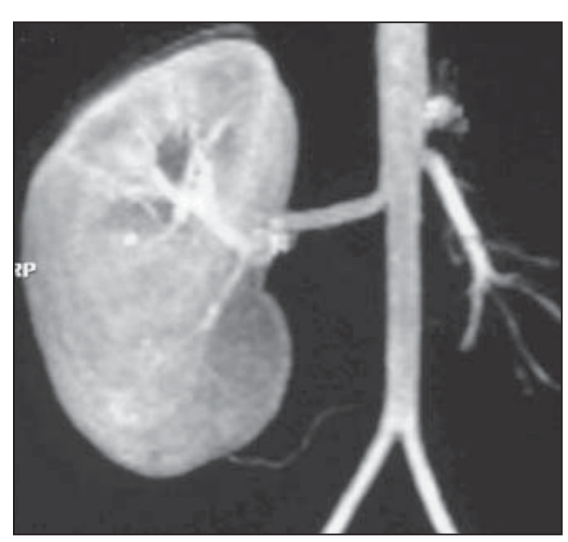

Figura 11. Agenesia renal esquerda.

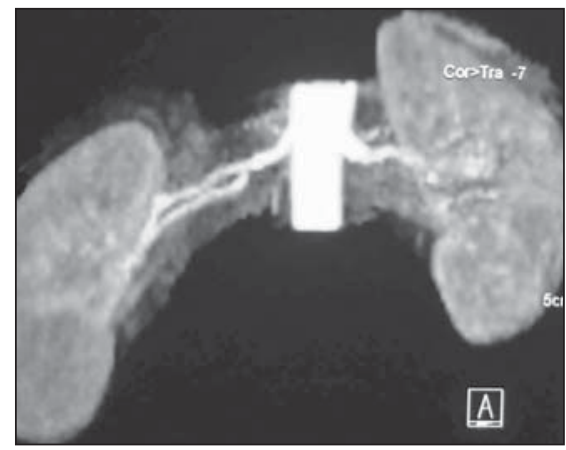

Figura 12. Bifurcação da artéria renal direita no seu terço médio.

\section{Considerações de imagem}

Dos 56 casos selecionados, $13(23,2 \%)$ foram considerados normais e 43 (76,7\%), alterados. Dentre os exames alterados, detectaram-se 22 estenoses de artéria renal $(20,1 \%), 20$ irregularidades parietais $(18,3 \%)$ nas artérias renais, 11 fluxos reduzidos (10\%) e cinco fluxos ausentes $(4,5 \%)$. Todos esses diagnósticos foram feitos com a técnica GE 3D após administração de gadolínio, concordando com os estudos de
Bongers et al..$^{(7)}$, Fain et $a l .^{(8)}$, Mittal et al. ${ }^{(9)}$ e Shetty et al. ${ }^{(\mathbf{1 0})}$. Com isso, consegue-se imagens de alta resolução dos principais ramos que compõem o sistema arterial renal, e pode-se correlacionar com as imagens no plano transversal. Por outro lado, ramos distais poderiam sofrer efeito de saturação devido ao seu menor calibre e fluxo reduzido, mimetizando certo grau de estenose $^{(11)}$.

\section{AORTA}

O estudo da aorta deve ser o primeiro tópico analisado no exame, visto que grande parte das indicações é por hipertensão arterial, que pode ser secundária a doença aterosclerótica difusa, promovendo estenose na origem da artéria renal. No nosso estudo, encontramos $44,66 \%(n=25)$ de aortas com irregularidades parietais secundárias a doença aterosclerótica.

A adequada visualização da aorta nos 56 casos estudados está de acordo com diversos estudos ${ }^{(10-14)}$. A obtenção das imagens em período de apnéia permitiu importante minimização dos artefatos respiratórios, concordando com o que foi observado nos trabalhos de De Cobelli et al. ${ }^{(\mathbf{1 5})}$, Shetty et al. ${ }^{(\mathbf{1 0})}$, Bongers et al. ${ }^{(7)}$ e Fain et al. ${ }^{(8)}$.

\section{ARTÉRIAS RENAIS E ESTENOSES}

A avaliação do trajeto das artérias renais, incluindo suas origens, foi considerada satisfatória em todos os casos, da mesma forma que nos estudos de Holland $e t$ al. ${ }^{(16)}$, Postma et al. ${ }^{(17)}$ e Rieumont et al. ${ }^{(12)}$.

Avaliamos 111 artérias renais e encontramos 19,81\% $(\mathrm{n}=22)$ de estenoses, sendo que $59,09 \%(n=13)$ localizavam-se no terço proximal e $31,81 \%(n=7)$, no terço médio. Isto pode ser explicado pelas faixas etárias estudadas e a associação com doença aterosclerótica. Por outro lado, tivemos 9,1\% $(\mathrm{n}=2)$ de estenoses no terço distal, que estavam relacionadas com a presença de displasia fibromuscular.

Devido à supervalorização das estenoses na angio-RM, muitos autores ${ }^{(7,9,13,17-19)}$ preconizam a avaliação das alterações hemodinâmicas e funcionais, em vez de se pesquisar apenas o grau da estenose arterial. Isto é importante, visto que o fator mais relevante na estenose é a existência ou não de alterações hemodinâmicas significativas, definindo se será ou não necessário algum procedimento de revascularização renal ${ }^{(9,17,18)}$.

No entanto, podem ser identificadas as estenoses hemodinamicamente significantes através da ausência de sinal no lugar da estenose. Também podem ser avaliadas as consequiências funcionais, incluindo a assimetria dos rins, diferença de impregnação do parênquima, excreção do contraste e perda da relação córtico-medular. Por isso, no nosso estudo, foram avaliados não só as artérias renais, mas também a aorta e os rins, para que se tentasse demonstrar essas alterações hemodinâmicas.

\section{RINS}

Apesar de o exame ser específico para as artérias renais, é importante que seja avaliada a função renal, dado este que pode ser identificado por sinais indiretos, relacionados ao realce do parênquima e à excreção do meio de contraste ${ }^{(13,19,20)}$.

Alguns autores ${ }^{(10,12,13,19)}$ defendem que o estudo da artéria renal deva ser, na verdade, um estudo dinâmico da perfusão do parênquima, tendo, dessa forma, o exame uma fase arterial, em que são estudadas as artérias, uma fase nefrográfica para o estudo do parênquima, uma fase venosa para o correto estudo da vascularização venosa e, finalmente, uma fase excretora, em que pode ser verificada a adequada eliminação do meio de contraste.

No nosso estudo foi observada boa correlação entre os achados de perfusão renal, como nefrogramas heterogêneos e até ausentes, com a presença de estenoses. No rim direito observamos 10,72\% $(n=6)$ de nefrogramas heterogêneos e no esquerdo, $12,74 \%(n=7)$. Ao correlacionar os nefrogramas com as estenoses da artéria renal 
direita, que foram de $14,28 \%(\mathrm{n}=8)$, e da esquerda, de $25,45 \%$ ( $n=14)$, observamos que quanto maior o grau de estenose, independentemente da topografia, maior a probabilidade de se encontrar alteração perfusional que poderia demarcar alteração hemodinamicamente significativas.

\section{CONSIDERAÇÕES FINAIS}

Podemos observar que a angio-RM tornou-se um método dinâmico e com capacidade diagnóstica diferenciada quando comparada aos outros métodos de exame. No nosso estudo identificamos artérias renais acessórias, fato importante na avaliação da vascularização renal e, principalmente, nos casos de avaliação pré-transplante. A angio-RM também permite a identificação de uma serie de outros achados, o que demonstra a capacidade diagnóstica do método.

O conhecimento dos artefatos e das limitações do método é de extrema importância na avaliação do exame, já que estes podem se tornar armadilhas diagnósticas.

A angiografia por subtração digital ainda é considerada como melhor método no estudo da anatomia vascular renal. No entanto, inúmeros trabalhos estão apontando o aumento da sensibilidade e da especificidade da angio-RM, principalmente após o surgimento de novas técnicas, mais rápidas e com melhores resoluções, o que faz deste exame um método completo, visto não ser invasivo, não utilizar radiações ionizantes, não utilizar contraste nefrotóxico e ter índices de complicações insignificantes quando comparado com a angiografia $\operatorname{digital}^{(7-11,14)}$.

\section{REFERÊNCIAS}

1. Claudon M, Plouin PF, Baxter GM, Rohban T, Devos DM. Renal arteries in patients at risk of renal arterial stenosis: multicenter evaluation of the echo-enhancer SH U 508A at color and spectral Doppler US. Levovist Renal Artery Stenosis Study Group. Radiology 2000;214:739-746.

2. Dong Q, Schoenberg SO, Carlos RC, et al. Diagnosis of renal vascular disease with MR angiography. RadioGraphics 1999;19:1535-1554.

3. Martin ML, Tay KH, Flak B, et al. Multidetector CT angiography of the aortoiliac system and lower extremities: a prospective comparison with digital subtraction angiography. AJR Am J Roentgenol 2003;180:1085-1091.

4. Tublin ME, Bude RO, Platt JF. Review. The resistive index in renal Doppler sonography: where do we stand? AJR Am J Roentgenol 2003;180: 885-892.

5. Gross CM, Krämer J, Weingärtner $\mathrm{O}$, et al. Determination of renal arterial stenosis severity: comparison of pressure gradient and vessel diameter. Radiology 2001;220:751-756.

6. Hernández-Hoyos M, Orkisz M, Puech P, Mansard-Desbleds C, Douek P, Magnin IE. Computerassisted analysis of three-dimensional MR angiograms. RadioGraphics 2002;22:421-436.

7. Bongers V, Bakker J, Beutler JJ, Beek FJ, De Klerk JM. Assessment of renal artery stenosis: comparison of captopril renography and gadolinium-enhanced breath-hold MR angiography. Clin Radiol 2000;55:346-353.

8. Fain SB, King BF, Breen JF, Kruger DG, Riederer SJ. High-spatial-resolution contrast-enhanced MR angiography of the renal arteries: a prospective comparison with digital subtraction angiography. Radiology 2001;218:481-490.

9. Mittal TK, Evans C, Perkins T, Wood AM. Renal arteriography using gadolinium enhanced 3D MR angiography - clinical experience with the technique, its limitations and pitfalls. Br J Radiol 2001;74:495-502.

10. Shetty AN, Bis KG, Kirsch M, Weintraub J, Laub
G. Contrast-enhanced breath-hold three-dimensional magnetic resonance angiography in the evaluation of renal arteries: optimization of technique and pitfalls. J Magn Reson Imaging 2000; 12:912-923.

11. Amaral LPG, Santos AASMD, Marchiori E. Angiografia por ressonância magnética do crânio: revisão de 100 casos. Radiol Bras 2004;37:153158.

12. Rieumont MJ, Kaufman JA, Geller SC, et al. Evaluation of renal artery stenosis with dynamic gadolinium-enhanced MR angiography. AJR Am J Roentgenol 1997;169:39-44.

13. Schoenberg SO, Knopp MV, Bock M, et al. Renal artery stenosis: grading of hemodynamic changes with cine phase-contrast MR blood flow measurements. Radiology 1997;203:45-53.

14. Völk M, Strotzer M, Lenhart M, et al. Time-resolved contrast-enhanced MR angiography of renal artery stenosis: diagnostic accuracy and interobserver variability. AJR Am J Roentgenol 2000; 174:1583-1588.

15. De Cobelli F, Venturini M, Vanzulli A, et al. Renal arterial stenosis: prospective comparison of color Doppler US and breath-hold, three-dimensional, dynamic, gadolinium-enhanced MR angiography. Radiology 2000;214:373-380.

16. Holland GA, Dougherty L, Carpenter JP, et al. Breath-hold ultrafast three-dimensional gadolinium-enhanced MR angiography of the aorta and the renal and other visceral abdominal arteries. AJR Am J Roentgenol 1996;166:971-981.

17. Postma CT, Joosten FBM, Rosenbusch G, Thien T. Magnetic resonance angiography has a high reliability in the detection of renal artery stenosis. Am J Hypertens 1997;10:957-963.

18. Debatin JF, Hany TF. MR-based assessment of vascular morphology and function. Eur Radiol 1998;8:528-539.

19. Hertz SM, Holland GA, Baum RA, Haskal ZJ, Carpenter JP. Evaluation of renal artery stenosis by magnetic resonance angiography. Am J Surg 1994;168:140-143.

20. Kroencke TJ, Wasser MN, Pattynama PM, et al Gadobenate dimeglumine-enhanced MR angiography of the abdominal aorta and renal arteries. AJR Am J Roentgenol 2002;179:1573-1582. 